\title{
GAMA: a new galaxy survey
}

\author{
I. Baldry,${ }^{1}$ J. Liske, ${ }^{2}$ S. P. Driver ${ }^{3}$ and the GAMA Team \\ ${ }^{1}$ Astrophysics Research Institute, Liverpool John Moores University, Twelve Quays House, \\ Egerton Wharf, Birkenhead CH41 1LD, UK \\ ${ }^{2}$ European Southern Observatory, Karl-Schwarzschild-Str. 2, 85748 Garching, Germany \\ ${ }^{3}$ SUPA, School of Physics \& Astronomy, University of St Andrews, St Andrews KY16 9SS, UK
}

\begin{abstract}
The case is outlined for a new galaxy survey, including spectroscopy with AAOmega and sub-arcsecond multi-band imaging, that bridges a crucial gap between the SDSS and VVDS surveys. The science focus is to study structure and the relationship between matter and light on kpc-to-Mpc scales. The range of scales probed will enable direct constraints on the Cold Dark Matter model by: (1) measuring the halo mass function down to $10^{12} \mathcal{M}_{\odot}$ and its evolution to $z \sim$ $0.4 ;(2)$ measuring the galaxy stellar mass function to very low mass limits of $10^{7} \mathcal{M}_{\odot}$ constraining baryonic feedback processes; and (3) quantifying the environment-dependent merger rate since $z \sim 0.4$. Here, we highlight the fact that the high-resolution imaging will enable the bulge-disk decomposition of $\sim 200000$ galaxies in $u-K$, providing a valuable resource for statistical studies of bulge properties.
\end{abstract}

Keywords. surveys, galaxies: bulges, galaxies: dwarf, galaxies: evolution, galaxies: fundamental parameters, galaxies: luminosity function, mass function, galaxies: stellar content, dark matter

International surveys, such as the Two-Degree Field Galaxy Redshift Survey (2dFGRS) and the Sloan Digital Sky Survey (SDSS) have transformed our view of large scale structure and have contributed directly towards the emergence of a concordance cosmology. These surveys have also provided a confirmation of the basic Cold Dark Matter (CDM) paradigm for the growth of structure through the comparison of robust model predictions with empirical clustering measurements on Mpc-to-Gpc scales. On smaller, sub-Mpc scales (i.e., on the scales of clusters, groups and galaxies) our theoretical understanding of the growth of structure is less well-founded and at kpc scales it breaks down almost entirely. It is on these scales (kpc-to-Mpc) where dark matter haloes virialize and merge, and where baryons decouple, collapse and eventually form complex structures such as galaxies. The kpc-to-Mpc range is therefore the key scale over which the baryons and baryon physics become critical to our understanding of the structures we see.

The Galaxy And Mass Assembly (GAMA) project aims to establish a definitive lowredshift survey of galaxies capable of testing CDM and the semi-analytic extensions that model the formation and evolution of galaxies over the $\mathrm{kpc}-\mathrm{Mpc}$ regime. That is the focus of the three top-level science goals listed in the abstract. Here, we highlight GAMA's capability at the lower end of the above scale regime to provide a comprehensive survey of the internal stellar structures of low-redshift galaxies.

GAMA will bring together high-resolution imaging in $u-i$ from $\mathrm{VST}$, in $Y-K$ from VISTA and spectroscopy from AAOmega, as well as HI and far-IR data, for $\sim 250000$ galaxies over $200 \mathrm{deg}^{2}$. The limiting factor is obviously the spectroscopy which sets the scope of the survey. The spectroscopic survey's selection function is preliminarily defined as $r<19.8$, or $K_{A B}<18.9$ with $r<20.5$. Recently, the GAMA project has been granted 66 nights with AAOmega on the Anglo-Australian Telescope, which should under reasonable conditions allow half the target spectra to be obtained. We initially aim at $50 \mathrm{deg}^{2}$ at the full target density with $100 \mathrm{deg}^{2}$ at a reduced magnitude limit. The 


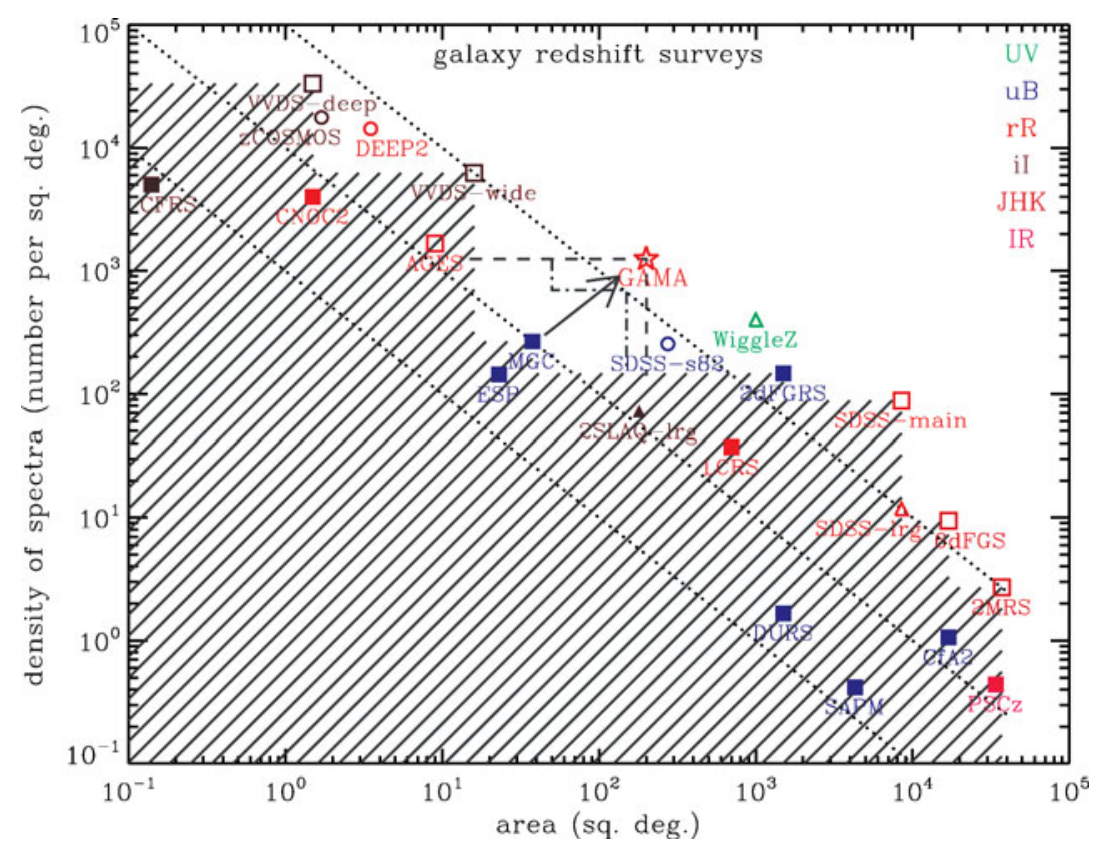

Figure 1. Comparison between galaxy redshift surveys: squares represent predominantly magnitude-limited surveys; circles represent surveys involving colour cuts for photometric redshift selection; while triangles represent highly targeted surveys. Filled symbols show completed surveys and the grey region shows the parameter space covered by magnitude-limited surveys. GAMA, shown by a star, cuts significantly into new parameter space. (The dash-and-dotted line represents the 3-year aim.) Surveys are colour coded according to selection wavelength. See www.astro.livjm.ac.uk/ ikb/research/galaxy-redshift-surveys.html for more details.

spectral resolution used will be $\sim 1300$ (over $370-880 \mathrm{~nm}$ ) allowing for velocity measurements with better than $50 \mathrm{~km} \mathrm{~s}^{-1}$ accuracy. Apart from redshifts the spectra will also yield classifications, stellar mass and age estimates, and reveal type 2 AGN.

Figure 1 shows a comparison between redshift surveys in terms of depth (defined using target density) versus area. GAMA builds a vital bridge between the shallow but large SDSS, and the deep but narrow VVDS-type surveys.

The imaging data will be of sufficient resolution and $\mathrm{S} / \mathrm{N}$ to allow the bulge-disk decomposition of the majority of our spectroscopic sample. Hence we expect to obtain high-quality measurements of structural parameters of the bulges and disks of $\sim 200000$ galaxies in $u-K$. These will be used for a number of statistical studies on the properties of bulges, including:

- A comprehensive determination of the bulge luminosity and stellar mass functions as a function of redshift ( to $z \approx 0.4$ ), environment, and $\mathrm{B} / \mathrm{T}$. The joint $r$ and $K$ selection probes total stellar masses of galaxies down to near $10^{7} \mathcal{M}_{\odot}$ at $z \geqslant 0.008$.

- Bulge demographics: a determination of the frequencies of red, high-Sérsic index (classical?) bulges and blue, low-Sérsic index (pseudo?) bulges, as a function of B/T and the properties of the disk which they 'inhabit'.

- A measurement of the colour gradients of bulges (using the multi-wavelength bulgedisk decompositions), again as a function of disk properties and redshift.

- A measurement of the joint stellar mass-size distribution of bulges, pseudo-bulges (and disks). 